\title{
INTELIGENCIA EMOCIONAL EN DISTINTOS COLECTIVOS: APORTACIONES DEL
}

\section{GRUPO TEAM+}

\author{
María del Pilar Berrios, Manuel Pulido-Martos, José María Augusto-Landa y Esther Lopez-Zafra \\ Universidad de Jaén, España
}

\section{Resumen}

En este artículo ofrecemos un resumen de los estudios que nuestro grupo de investigación TEAM ${ }^{+}$ha llevado a cabo a lo largo de 16 años. Durante todos estos años hemos analizado, desde una perspectiva psicosocial, los efectos y beneficios de la inteligencia emocional en diferentes ámbitos y colectivos. Consideramos que lo psicosocial es central en el análisis de la inteligencia emocional puesto que las personas se encuentran la mayor parte de su tiempo en un contexto y en relación con otras personas. Por ello, nos interesa conocer cómo la inteligencia emocional contribuye al logro de resultados positivos (p.ej., bienestar, satisfacción o rendimiento) y promueve entornos más saludables con menos riesgos psicosociales (p.ej., estrés, burnout o depresión). Nuestros trabajos confirman que la IE actúa como un recurso personal que amortigua los efectos negativos del estrés y del burnout en colectivos con especial incidencia, como el de enfermería y profesorado. Asimismo, la IE mejora diferentes indicadores del bienestar subjetivo de estos profesionales y de trabajadores de otros contextos laborales. Es decir, por un lado, la inteligencia emocional se muestra como un factor facilitador de resultados saludables: satisfacción laboral, satisfacción vital, bienestar psicológico, mejores relaciones sociales y/o de pareja, mejor rendimiento; y por otro, como un factor protector de diferentes riesgos: estrés, burnout, dolor en la enfermedad o relaciones de pareja violentas. Estos efectos no solo ocurren a nivel individual, sino que también afectan a los procesos grupales, como el ejercicio del liderazgo en diferentes contextos. Por todo ello, es necesario incrementar los niveles de inteligencia emocional de la población en distintos contextos con programas adaptados a la realidad específica que les permita hacer frente a las demandas y retos del entorno social y laboral.

Palabras clave: Inteligencia emocional, Adolescencia, Liderazgo, Enfermería, Docentes, Grupos vulnerables.

\section{Abstract}

In this paper we summarize the studies that our $\mathrm{TEAM}^{+}$research group has carried out over 16 years. Along these years we have analyzed, from a psychosocial perspective, the effects and benefits of emotional intelligence in different areas and groups. We consider that the psychosocial aspect is central in the analysis of emotional intelligence since people are most of their time in a context and in relation to other people. Therefore, we are interested in knowing how emotional intelligence contributes to the achievement of positive results (i.e. well-being, satisfaction or performance) and promotes healthier environments with fewer psychosocial risks (i.e. stress, burnout or depression). Our results confirm the hypothesis that emotional intelligence acts as a personal resource that buffers the negative effects of stress and burnout in groups with special incidence, such as nursing and teaching staff. Likewise, emotional intelligence improves different indicators of the subjective well-being of workers from different work contexts. That is, on the one hand, emotional intelligence is shown as a facilitating factor for healthy results: job satisfaction, life satisfaction, psychological well-being, better social and / or partner relationships, better performance; and on the other, as a protective factor against different risks: stress, burnout, pain in the disease or violent relationships. These effects not only occur at the individual level, but also affect group processes, such as leadership in different contexts. Therefore, it is necessary to increase the emotional intelligence levels of the population in different contexts with programs adapted to the specific reality that allow them to face the demands and challenges of the social and work environment.

Keywords: Emotional intelligence, Adolescence, Leadership, Nursing, Teachers, Vulnerable groups.
Esther Lopez-Zafra

elopez@ujaen.es

Departamento de Psicología

Universidad de Jaén 


\section{Introducción}

Desde que Salovey y Mayer (1990) publicaron la primera definición operativa del constructo de inteligencia emocional (IE), se han desarrollado distintos modelos teóricos, instrumentos de medida y programas de intervención destinados a mejorar las habilidades socio-emocionales de las personas. Estos avances han sido publicados en numerosas revistas científicas nacionales e internacionales de reconocido prestigio. Además, en nuestro país se han publicado monográficos o números especiales sobre IE en revistas españolas como Psicothema, Ansiedad y Estrés, Electronic Journal of Research in Educational, y Behavioral Psychology / Psicología Conductual, que han recogido los avances conseguidos y retos planteados en este campo de investigación. Este monográfico se suma a las iniciativas anteriores para dar nuevas respuestas y/o consolidar respuestas ya conocidas acerca de los mecanismos a través de los cuales la IE influye en la toma de decisiones y las acciones que llevan al éxito y el logro de objetivos. La cantidad de publicaciones científicas que existen ponen de manifiesto la relevancia del tema, el interés que suscita y la gran aplicación que tiene en diferentes ámbitos como la salud, la educación y el trabajo.

En este artículo ofrecemos un resumen de los estudios que nuestro grupo de investigación $\mathrm{TEAM}^{+}$ha llevado a cabo a lo largo de 16 años. Durante todos estos años hemos analizado, desde una perspectiva psicosocial, los efectos y beneficios de la IE en diferentes ámbitos y colectivos. En nuestra investigación tenemos en cuenta que la IE puede medirse, bien a través del meta-conocimiento que tienen las personas sobre su propia IE, esto es inteligencia emocional percibida (IEP, o rendimiento típico autoinformado) o mediante pruebas de rendimiento máximo (o de habilidad) (MacCann et al., 2014). En ambos casos se evalúan diferentes dimensiones de la IE, es decir, habilidades socioemocionales percibidas u objetivas como la percepción, facilitación, comprensión o regulación emocional.

Nuestros trabajos se basan en la idea de que lo psicosocial es central en el análisis de la IE puesto que las personas se encuentran la mayor parte de su tiempo en un contexto y en relación con otras personas. Por ello, nos interesa conocer cómo la IE contribuye al logro de resultados positivos (p.ej., bienestar, satisfacción o rendimiento) y promueve entornos más saludables con menos riesgos psicosociales (p.ej., estrés, burnout o depresión). Por ejemplo, hemos estudiado el papel de la IE en el ámbito laboral y los procesos de liderazgo porque las relaciones humanas son cruciales en el trabajo, y solo cuando el líder es capaz de reconocer, etiquetar y comprender sus emociones (y las de los demás), puede elegir las formas más útiles de expresarlas y regularlas, y ayudar a los miembros de su equipo a expresar y regular las suyas. También nos hemos centrado en colectivos profesionales como los enfermeros/as y los docentes, por tratarse de profesiones asistenciales de alta responsabilidad social expuestas a altas demandas intelectuales y emocionales que hacen que estos profesionales tengan más riesgo de experimentar estrés y los problemas emocionales derivados del mismo (ansiedad, depresión, síndrome de burnout...). Además, hemos analizado la importancia de la IE en los jóvenes y adolescentes porque son un colectivo que se encuentra en una etapa de muchos cambios sociales, contextuales y personales; y las habilidades socioemocionales que desarrollen pueden protegerlos de las situaciones de riesgo que se les presenten, y ayudarlos a orientar sus conductas hacia el logro de metas y objetivos. Por último, también hemos investigado cómo la IE puede ayudar a diferentes grupos de alta vulnerabilidad social (mujeres víctimas de violencia de género, inmigrantes, desempleados, enfermos crónicos o mayores institucionalizados) a sobreponerse a los efectos negativos de las adversidades que tienen que afrontar y a desarrollar su potencial para lograr resultados positivos en sus vidas.

A continuación, se resumen los principales resultados de todos estos estudios y se analizan sus principales implicaciones prácticas desde un punto de vista individual y social, con el fin de hacer aportaciones que contribuyan a realizar los cambios necesarios para conseguir que las personas emocionalmente inteligentes construyan sociedades emocionalmente inteligentes.

\section{El papel de la inteligencia emocional en el trabajo y en los procesos de liderazgo}

En el ámbito organizacional y laboral, el análisis de los efectos de la IE sobre diferentes resultados ha generado mucho interés, no solo en el nivel individual sino también en los niveles grupal y organizacional (para una revisión véase Lopez-Zafra y Pulido-Martos, 2020).

La IE influye o actúa como mediador y/o moderador en diferentes resultados positivos: objetivos como el rendimiento o eficacia (Ayiro, 2014) y subjetivos como la satisfacción o felicidad de los trabajadores (Lopes, 2016). Su influencia puede darse a distintos niveles (Ashkanasy y Dorris, 2017), por lo que hay que considerar, entre otros, el análisis multinivel y su impacto en la creación de organizaciones saludables (Salanova, et al., 2019) o el efecto que la IE puede tener en algunos procesos grupales específicos como el liderazgo. Precisamente, los procesos de liderazgo se han analizado desde una perspectiva multi-nivel y es uno de los temas que mayor atención ha generado en el ámbito laboral. $\mathrm{Su}$ efecto en los resultados organizacionales es evidente. Un buen líder puede mejorar los resultados del grupo, pero un liderazgo inadecuado puede perjudicar el rendimiento, la satisfacción y hacer que los trabajadores deseen abandonar su trabajo. La IE puede ser la responsable de estos resultados (Cherniss y Roche, 2020). De hecho, una de las variables que se ha comprobado que media 
la relación entre el liderazgo, sobre todo estilos de liderazgo positivos, y diferentes resultados de trabajo, es la IE (Lord et al., 2017). La investigación muestra que la IE del líder influye en la satisfacción laboral de los miembros de su grupo (Miao et al., 2016). En cuanto a los diferentes estilos de liderazgo, en la última década destacan las contribuciones del liderazgo transformacional (Kramer et al., 2019). Los líderes transformacionales aumentan la satisfacción de los seguidores, sus comportamientos de ciudadanía organizacional y sus niveles de rendimiento (Braun et al., 2013). Es decir, se produce un efecto positivo sobre variables individuales y grupales como son el compromiso de los empleados, la motivación y la ejecución eficiente de tareas. Esta perspectiva sugiere que los líderes efectivos son aquellos cuyas cualidades personales les capacitan para articular una visión que logran transmitir a un grupo determinado. En definitiva, se enfatiza la importancia del líder, pero también de los miembros del grupo que lidera y dirige.

\section{Resultados y aportaciones del grupo TEAM ${ }^{+}$}

Nuestro equipo ha analizado el impacto de la IE en distintos niveles de análisis. En el nivel individual, se comprueba que elementos de la IE (p.ej., regulación emocional) explican algunas características individuales o intrapersonales de los trabajadores (p.ej., empatía), con efectos positivos en distintos aspectos del funcionamiento personal, social y laboral. En este sentido, nuestro trabajo muestra precisamente que la IE produce una validez incremental sobre la empatía en la explicación de la eficacia social, es decir, aumenta el éxito de las relaciones sociales en el trabajo (Berrios et al., 2013). Desde el punto de vista individual, se considera que las mujeres son más empáticas y también tienen mayores niveles de IE, si bien nuestros estudios muestran que esto tiene más que ver con la dimensión evaluada y el modelo de IE empleado (véase Lopez-Zafra y Gartzia, 2014). Sin embargo, en el estudio de Berrios et al., esta eficacia social es independiente del género. Es decir, la IE ayuda a explicar variables individuales que son eficaces a nivel grupal, como las relaciones o procesos de interrelación. Un caso concreto en el que este hecho tiene implicaciones laborales relevantes es en la negociación, que comprende un nivel de análisis interpersonal. Analizamos el papel de la IE durante los procesos de negociación teniendo en cuenta el control de las variables de personalidad, para comprobar si la percepción de habilidades emocionales puede explicar los resultados obtenidos (Pulido-Martos et al., 2013). La regulación emocional resulta clave en la efectividad de la negociación, independientemente de las características de personalidad, mostrando que los negociadores con mayores niveles de regulación emocional crean un ambiente donde no se percibe el desequilibrio de poder. Nuestros resultados apoyan el peso de la regulación emocional en muchos procesos de relaciones interpersonales en el trabajo.
Esta capacidad de regular emociones y contar con niveles elevados de IE es muy relevante en el caso de directivos y mandos intermedios puesto que afecta a sus niveles de salud mental y percepción de estrés. En concreto, comprobamos que se produce una relación positiva entre IEP y salud mental (Luque-Reca et al., 2014) y que los niveles de estrés percibido median la relación entre la claridad y reparación emocional y los niveles de salud mental. Nuestro estudio muestra que las habilidades emocionales facilitan un mejor afrontamiento ante las reacciones negativas del entorno laboral en una población con la que no se había trabajado antes: directivos y mandos intermedios.

Precisamente los grupos de trabajo están constituidos por personas cuya forma de liderar afecta al funcionamiento del grupo, por lo que el análisis del estilo de liderazgo de los directivos podría tener una relación concreta con la IE. Publicamos un primer trabajo en el que se comprobaba empíricamente esta relación, y se analizaba la emergencia del liderazgo considerando las habilidades emocionales (Lopez -Zafra, Garcia-Retamero et al., 2008). Analizamos si las personas con niveles más altos de IE emergen con mayor frecuencia como líderes debido a un estilo más transformacional. Además, tuvimos en cuenta si la cohesión grupal afectaba a esa relación. Los resultados muestran que las personas con mayores niveles de claridad y regulación emocional, son elegidas y, por tanto, emergen como líderes, pero solo cuando manifiestan un estilo de liderazgo transformacional, en grupos cohesivos. Nos preguntamos si, además, el sexo de la persona que ejerce el liderazgo puede influir en esta relación. En este sentido, analizamos si la relación que se produce entre IE y liderazgo transformacional, que ya había sido destacada en varios estudios, se ve afectada por el género (Lopez-Zafra et al., 2012). Esta idea surge por los resultados de distintas investigaciones en las que se propone, por una parte, que la IE es más elevada en mujeres y, por otro lado, que el estilo de liderazgo transformacional se relaciona con características típicas del estereotipo femenino, es decir, más comunales o de orientación hacia el grupo. Sin embargo, los análisis parciales de estas relaciones sugieren que no es tan relevante el sexo como el género, es decir, las características asociadas al género que las personas asumen. Los resultados muestran que los niveles individuales de IE y de roles de género predicen el estilo de liderazgo transformacional, independientemente del sexo. Sin embargo, se producen importantes diferencias entre las titulaciones en las que se realizó el análisis. En concreto, si eran estudiantes de carreras típicamente femeninas o neutrales (p.ej., psicología o económicas) sus puntuaciones eran superiores en atención a las emociones y feminidad, que si estudiaban en una titulación típicamente masculina (p.ej., ingeniería). Por contra, si estudiaban titulaciones neutrales (p.ej., económicas) o típicamente masculinas, puntuaban más en regulación emocional que en una titulación típicamente fémina. Este resultado apoya el he- 
cho de que las personas que eligen disciplinas con tipicidad de género puntúan las características relacionadas con ese género, independientemente de su sexo.

Para dar un paso más y analizar estas cuestiones con trabajadores, teniendo en cuenta el nivel de análisis grupal, comprobamos que los miembros de un grupo tienen percepciones similares, tanto del estilo de liderazgo de sus líderes como de la IE de sus grupos (Lopez-Zafra et al., 2017). Es decir, se demuestra que el liderazgo transformacional y la IE grupal están relacionados a nivel grupal y que el liderazgo transformacional es uno de los predictores de la percepción de la IE grupal en entornos laborales.

\section{EI papel de la IE en profesionales de enfermería}

El burnout constituye un serio problema para el colectivo de enfermería (Li et al., 2018), teniendo como responsables estresores ocupacionales. Estos estresores son: el contacto con el sufrimiento y la muerte, los conflictos con los compañeros, la falta de preparación para tratar las necesidades emocionales de pacientes y familiares, la incertidumbre acerca de la eficacia del tratamiento, el cansancio y fatiga, el miedo a incurrir en negligencias o impericias y el trabajo nocturno (Blanco, 2004; McTiernan y McDonald, 2014). Los trabajos realizados en el ámbito de las competencias emocionales han proporcionado evidencias de que la IE podría moderar la relación entre el estrés y el agotamiento. Esto no es sorprendente ya que la IE promueve un mejor manejo de situaciones negativas o estresantes. La investigación con estos profesionales muestra que las personas con elevada IE tienen más probabilidades de evaluar las situaciones estresantes como un desafío más que como una amenaza, y tienen más confianza en que pueden hacer frente a tales situaciones (Görgens-Ekermans y Brand, 2012; Hong y Lee, 2016; Szczygiel y Mikolajczak, 2018).

En cuanto a los trabajos que han puesto en relación la IE con la satisfacción laboral y salud, apuntan en términos generales que la IE se relaciona positivamente con la salud y satisfacción laboral y negativamente con el estrés/ burnout. Así, en el contexto de la enfermería contamos con trabajos que encuentran relaciones positivas entre IE y satisfacción laboral y sugieren que la IE puede mejorar la salud general, satisfacción laboral y disminuir el agotamiento de este colectivo (Cummings et al., 2005; Powell et al., 2015; Soto-Rubio et al., 2020).

Por tanto, podemos afirmar que las emociones juegan un papel fundamental en el colectivo de la enfermería, ya que la capacidad de razonar sobre ellas, percibirlas y comprenderlas permitirá a las personas desarrollar procesos de regulación emocional que ayudarán a modelar y prevenir los efectos negativos del estrés (Schmitz et al., 2009).

\section{Resultados y aportaciones del grupo TEAM}

Los estudios que hemos realizado con nuestro equipo en el ámbito de los profesionales de enfermería han puesto en relación la IE, y en concreto la IEP, con variables tales como, el burnout, estrés ocupacional, salud, bienestar psicológico, satisfacción laboral y la calidad de vida. Así, investigamos en una muestra de enfermeros la relación entre IEP, burnout y salud mental (Augusto-Landa et al., 2006). Los resultados encontrados muestran que una alta atención emocional explica los componentes de burnout fatiga emocional y despersonalización. Por otra parte, una adecuada atención emocional y una alta compresión y regulación emocional explican el componente de realización personal. Con respecto a la salud mental una baja atención emocional $\mathrm{y}$ alta comprensión y regulación explican mejores resultados.

En esta misma línea, investigamos la relación entre IEP, estresores ocupacionales y salud en estos profesionales (Augusto-Landa et al., 2008). Nuestros resultados muestran que los componentes de la IEP explican la percepción de estresores ocupacionales. En concreto, los sujetos con alta claridad y reparación emocional informan de menor estrés, mientras que aquellos sujetos con alta atención a sus emociones experimentan mayor estrés. Así los componentes de IEP, claridad y reparación emocional se constituyen como factores protectores frente al estrés y facilitadores de la salud.

Analizamos también la relación entre IEP y satisfacción laboral en un grupo de enfermeras profesionales de un hospital de Jaén (Berrios et al., 2006). Los resultados muestran que la satisfacción laboral general es explicada por los niveles de reparación emocional. Estos resultados apuntan que aquellas personas con mayor capacidad de regulación de sus propias emociones, informan de mayor satisfacción laboral. Este factor tiene un gran peso específico al relacionarse con actitudes positivas hacia el trabajo.

En otros estudios hemos investigado la relación entre la IEP y el bienestar psicológico y calidad de vida en profesionales de enfermería. Encontramos que la calidad de vida es explicada por el factor reparación emocional, de tal manera que las enfermeras con alta reparación emocional informan de mayor calidad de vida (Montes-Berges y Augusto-Landa, 2014). Con estudiantes de último curso de la titulación de enfermería, analizamos la relación entre IEP y bienestar psicológico (Pulido-Martos, Augusto-Landa et al., 2016), encontrando que la reparación emocional consigue explicar algunos de los componentes del bienestar psicológico (p.ej., auto-aceptación, relaciones positivas, dominio del entorno y propósito de vida). Otras dimensiones del bienestar, como autonomía y crecimiento personal, eran explicadas por los niveles de claridad y atención emocional. 


\section{El papel de la IE en los docentes}

En la nueva realidad educativa los docentes están sometidos a altas responsabilidades y exigencias, y a nuevas demandas y retos. Ahora no es suficiente conseguir un buen rendimiento académico del alumnado, sino que además los profesores tienen la responsabilidad de facilitar y potenciar el desarrollo integral del estudiantado, su talento y creatividad (Cabello et al., 2010). Para hacer frente a estos objetivos el docente no solamente debe prepararse en nuevas materias, sino que además ha de trabajar en equipo, coordinarse por niveles, ciclos y materias, asumir en algunos casos cargos de gestión (Director, Jefe de Estudios, Secretario...), y pertenecer a órganos colegiados (Claustro de profesores, Consejo Escolar, Comisiones...). Estas responsabilidades, junto con las elevadas demandas por parte de alumnos y padres, y las continuas reformas de los planes de estudio, están convirtiendo la docencia en un trabajo de alto riesgo psicosocial (Salanova et al., 2003). En este escenario, estos profesionales se exponen a numerosas demandas complejas, intensas y en muchos casos contradictorias, que afectan tanto a su eficacia profesional como a su equilibrio y desarrollo humano (Peña et al., 2018). Así que, podemos concluir que la profesión docente es retadora y de alta responsabilidad social, pero al mismo tiempo estresante (Hargreaves, 2000; Moriana y Herruzo, 2004).

El agotamiento de los docentes puede afectar negativamente al funcionamiento del aula al disminuir las interacciones positivas con los estudiantes y/o proporcionarles feedback negativo sobre su rendimiento (Travers, 2001). La literatura científica señala que el desarrollo de habilidades socioemocionales permite a los profesores lidiar con los conflictos, disminuir sus niveles de estrés y agotamiento (Extremera et al., 2003; Pena y Extremera, 2010; Puertas Molero et al., 2019), construir relaciones positivas, mejorar su desempeño y clima en el aula (Brackett et al. 2010; Carson et al., 2011; Sutton, 2004), y experimentar engagement en su trabajo (Pena y Extremera, 2010) obteniendo así mejores resultados en el proceso enseñanza-aprendizaje (mejor desarrollo socio-emocional y rendimiento de sus alumnos) (Brackett et al., 2012; Greenberg et al., 2003; Hagelskamp et al., 2013; Rivers et al., 2013).

\section{Resultados y aportaciones del grupo TEAM ${ }^{+}$}

Tal y como hemos visto en el apartado anterior, la literatura científica indica que uno de los principales recursos personales que pueden utilizar los profesores para afrontar los principales obstáculos y demandas de su trabajo, y mejorar su rendimiento y desarrollo personal es la IE. De hecho, contamos con evidencias empíricas sólidas y robustas que confirman que la IE es un recurso personal que: 1) amortigua/protege a los docentes de los principales riesgos psicosociales de su trabajo (estrés y burnout), y 2) facilita/ potencia su motivación, rendimiento y bienestar laboral (engagement, mejores resultados en el proceso enseñanzaaprendizaje y satisfacción). Nuestro equipo de investigación ha llevado a cabo diferentes investigaciones para comprobar si se constatan estas relaciones.

Por ejemplo, realizamos un estudio con profesores de educación primaria para analizar las relaciones entre la IEP, el estrés y la salud mental de los docentes. Por una parte, encontramos una relación negativa entre claridad emocional, rol emocional y función social. De forma que, en los maestros con mayor claridad emocional, se comprueba una menor influencia de los problemas emocionales en el trabajo y las actividades sociales. Por otra parte, los resultados también indican que el impacto que tienen en el trabajo los problemas emocionales derivados de sus principales fuentes de estrés (p.ej., altas exigencias laborales y necesidad de adaptación a los continuos cambios) depende de habilidades de la IEP como la atención y la claridad emocional. Concretamente, la relación entre las exigencias laborales y los problemas emocionales es más positiva en los docentes con alta atención emocional, y la relación entre la adaptación a los cambios y los problemas emocionales es más negativa en los docentes con alta atención y baja claridad emocional. De acuerdo con estos resultados, concluimos que la IEP es un moderador importante de la relación entre los estresores ocupacionales de los profesores y su salud mental (Pulido-Martos, Lopez-Zafra et al., 2016).

En otro estudio investigamos la relación entre IEP, el afecto positivo y negativo, y el síndrome de burnout en maestros de educación primaria, y encontramos que las habilidades intrapersonales de la IEP (atención, claridad y reparación emocional) se relacionan positivamente con el afecto positivo y con la realización personal. Además, analizamos las vías de influencia de las habilidades de la IEP en el afecto positivo y negativo, y sus efectos en el síndrome de burnout, y comprobamos que la atención emocional y el afecto negativo influyen directamente en las dimensiones del síndrome de burnout. Los docentes que atienden más a sus emociones experimentan más cansancio emocional y más realización personal, y los que experimentan más afecto negativo experimentan más cansancio emocional, más despersonalización y se sienten menos realizados en su trabajo. Por otra parte, los principales efectos indirectos ponen de manifiesto que tanto la atención como la reparación emocional previenen/amortiguan los síntomas del síndrome de burnout a través del afecto positivo. De forma que, la atención y reparación emocional influyen positivamente en el afecto positivo, y éste influye negativamente en los niveles de burnout. Estos resultados nos llevan a concluir que las habilidades de la IEP explican por sí mismas una parte importante del burnout, y que todas las variables incluidas en el modelo (atención, claridad y reparación emocional, afecto positivo y afecto negativo) explican el $80 \%$ de este síndrome (Augusto-Landa et al., 2012). 
Además, también pudimos constatar con maestros de educación primaria que los docentes con más claridad emocional utilizan estrategias conductuales (p.ej., planificación y puesta en marcha de acciones para eliminar/superar el estresor o solucionar situación), cognitivas (i. e. reinterpretación de la situación para manejarla y/o solucionarla adecuadamente o para que deje de generar malestar) y/o centradas en las emociones (p.ej., tendencia a centrarse en el malestar experimentado en la situación y compartir con otras personas dicha situación y las emociones negativas); mientras que los docentes con mayores niveles de reparación emocional utilizan más las estrategias cognitivas y las centradas en las emociones, no son demasiado sensibles a la desaprobación de los demás y se preocupan menos por los sucesos que escapan a su control. Además, ni la claridad ni la reparación emocional se relacionan con las estrategias de escape cognitivo (evitar pensar en la situación que causa estrés) y escape conductual (consumo de alcohol y drogas). Estos resultados nos llevan a concluir que los profesores con más habilidades para comprender y regular sus emociones manejan mejor las situaciones estresantes utilizando estrategias de afrontamiento activas y adaptativas (AugustoLanda et al., 2011).

Por último, analizamos las relaciones entre IEP, satisfacción vital y satisfacción laboral en profesores universitarios. Los resultados indican que los docentes con más IEP están más satisfechos con su vida, y que la claridad y reparación emocional explican una pequeña pero significativa parte de la satisfacción vital no explicada por otras variables como los rasgos de personalidad y el estado de ánimo (Augusto-Landa et al., 2006).

\section{El papel de la IE en los jóvenes y adolescentes}

La sociedad actual tiene la responsabilidad de buscar nuevas formas de educar que promuevan el crecimiento saludable de los estudiantes, y potencien al mismo tiempo el talento y la creatividad (Berrios et al., 2016; Clouder et al., 2008). Ante este reto, las investigaciones realizadas en el ámbito educativo en los últimos 30 años han permitido identificar las habilidades socio-emocionales necesarias para el desarrollo integral del ser humano. La evidencia empírica acumulada en el ámbito educativo indica: 1) que la IE favorece la salud mental, previene las conductas de riesgo, mejora la empatía, el ajuste psicosocial, el bienestar y el rendimiento académico de los estudiantes (Extremera y Fernández-Berrocal, 2003; Fernández-Berrocal y Extremera, 2010; Van Heck y Den Oudsten, 2008); y 2) que a través de la práctica y el entrenamiento se pueden perfeccionar las habilidades socio-emocionales en diferentes situaciones de la vida cotidiana (Fernández-Berrocal y Extremera, 2005; Mayer et al., 2008).

\section{Resultados y aportaciones del grupo TEAM ${ }^{+}$}

De acuerdo con todo lo anterior, nuestro equipo ha llevado a cabo diferentes investigaciones para comprobar si se constatan estas relaciones. Por ejemplo, llevamos a cabo una investigación con estudiantes de enfermería de la Universidad de Jaén cuyos resultados pusieron de manifiesto que los estudiantes con más atención emocional buscan más apoyo social, los de mayor claridad emocional identifican mejor las emociones asociadas a las situaciones de estrés, e invierten menos tiempo en atenderlas para usar correctamente sus recursos cognitivos en estrategias de afrontamiento adaptativas; y los que tienen más habilidad para reparar sus emociones buscan más apoyo social y tienen una mejor salud mental (Montes-Berges y Augusto-Landa, 2007).

En cuanto a la relación de la IE con diferentes indicadores de bienestar, hemos llevado a cabo estudios centrados tanto en el bienestar subjetivo de los estudiantes (p. ej. satisfacción con la vida) como en su bienestar psicológico. En relación con el primero, hemos comprobado que los estudiantes universitarios con más claridad emocional y más habilidad para regular sus sentimientos informan de mayores niveles de satisfacción con la vida, y que los factores que mejor explican este componente del bienestar subjetivo son el afecto positivo y la claridad emocional (Augusto-Landa et al., 2004). En esta misma línea, también hemos encontrado en estudiantes de secundaria que la IEP influye positivamente en su satisfacción con la vida de manera indirecta a través del apoyo social. Es decir, los alumnos con más IEP tienen más apoyo social de su familia, amigos y centro educativo, lo que a su vez influye positivamente en su satisfacción con la vida y negativamente en sus niveles de depresión. Además, la interacción de la IEP con el apoyo social también influye positivamente en la satisfacción con la vida y negativamente en la depresión (Lopez-Zafra et al., 2019). Por último, en lo que al bienestar subjetivo se refiere, evaluando la IE con una prueba objetiva, obtuvimos resultados según los cuales la habilidad para manejar las emociones propias, junto con el sentido del humor, explican un porcentaje importante del bienestar subjetivo de los estudiantes (Berrios et al., 2012).

Por otra parte, en cuanto al bienestar psicológico de los estudiantes, hemos encontrado que los universitarios más extrovertidos y menos neuróticos registran mayores niveles de bienestar psicológico (Augusto-Landa et al., 2010). Ahora bien, una vez controlado el efecto de los rasgos de personalidad, las habilidades de la IEP (atención, claridad y reparación emocional) explican una parte significativa de los componentes del bienestar psicológico (autoaceptación, relaciones positivas, autonomía, competencia, crecimiento y desarrollo personal, y vida con sentido). En esta misma línea de investigación, también hemos comprobado que otro rasgo de personalidad como el optimismo y los com- 
ponentes de la IEP se relacionan positivamente con los factores del bienestar psicológico, mientras que el pesimismo está negativamente asociado a dichos factores (Augusto -Landa et al., 2011). Analizando las vías de influencia de la IEP en el optimismo/pesimismo y sus efectos en el bienestar psicológico, encontramos que la claridad y reparación emocional ejercen una influencia directa e indirecta, a través del optimismo y pesimismo disposicional, en todos los componentes de bienestar psicológico. De esta forma, los estudiantes con más habilidades para comprender y reparar sus emociones son más optimistas y menos pesimistas, lo que a su vez influye positivamente en su bienestar psicológico.

En lo que se refiere al ajuste social y la autoestima de los jóvenes y adolescentes observamos, por un lado, que la IEP explica una parte significativa del autoconcepto y la autoestima de los jóvenes universitarios más allá de lo que explican los rasgos de personalidad (Augusto-Landa et al., 2009); y por otro, que los estudiantes con altos niveles de IEP muestran más actitudes prosociales relacionadas con la competencia social (ayuda y colaboración, sensibilidad social, seguridad y firmeza, y liderazgo prosocial), tienden a ser empáticos y cooperadores con sus compañeros; y son valorados por sus profesores como estudiantes mejor adaptados socialmente (Jiménez y Lopez-Zafra, 2011).

Además, hemos realizado estudios sobre la relación de la IE con el rendimiento académico, comprobando que la IEP de los estudiantes ejerce una influencia positiva y directa en el rendimiento, y una influencia indirecta a través del manejo de la ansiedad. Esto es, los estudiantes con más IEP experimentan menos ansiedad, lo que a su vez favorece su rendimiento (Cortés-Denia et al., 2020). No obstante, también hemos constatado que la relación entre estas variables depende del tipo de medida utilizada para evaluar la IE. En un meta-análisis que llevamos a cabo con estudiantes de secundaria (Sánchez-Álvarez et al., 2020) analizamos las relaciones entre la IE y el rendimiento teniendo en cuenta los diferentes instrumentos desarrollados desde los distintos enfoques de la IE, y los resultados confirmaron un efecto significativo moderado de la IE (evaluada desde tres enfoques diferentes) en el rendimiento académico. Además, encontramos diferencias en los niveles de asociación entre la IE y el rendimiento en función del tipo de medida. La relación entre estas variables es mayor cuando se evalúa la IE con pruebas objetivas. A pesar de que en el contexto académico se utilizan más las medidas subjetivas de IE, cuando se utilizan este tipo de medidas los efectos de la IEP en el rendimiento no están claros, ya que se pueden superponer los efectos de las habilidades socioemocionales con los efectos de características de personalidad. Por último, también encontramos gran heterogeneidad en los resultados dentro de cada instrumento. Esto puede deberse a la influencia de variables como la edad, el sexo, el CI, la personalidad de los estudiantes... o a las variaciones en las adaptaciones de los instrumentos a diferentes idiomas. Todo esto nos llevó a concluir que el origen de todas las fuentes de heterogeneidad es el uso de diferentes instrumentos, y para resolver esta falta de consenso sería necesario que se usen instrumentos con buenas propiedades psicométricas y una sólida trayectoria.

\section{El papel de la inteligencia emocional en la vulnerabili- dad social y resiliencia}

La vulnerabilidad es un constructo abordado desde múltiples disciplinas que, además de abarcar la exposición individual a riesgos de tipo físico, psicológico y/o emocional (Morese et al., 2019), incorpora una dimensión social (se habla así de vulnerabilidad social) que se refiere a la incapacidad de personas, grupos y comunidades para sobreponerse a los efectos negativos de diferentes estresores, no llegando a poder desarrollar todo su potencial ni alcanzar resultados de tipo positivo en sus vidas (Burton et al., 2018; Loughhead y Mittai, 2000). Desde esta perspectiva es posible identificar grupos vulnerables como mujeres víctimas de violencia de género, inmigrantes, desempleados, enfermos crónicos o mayores institucionalizados, entre otros, que resultan de la exposición a factores de riesgo como el género, el estatus social, el nivel de ingresos, los niveles de salud o la edad (Burton et al., 2018; Cutter et al., 2003).

Aunque la vulnerabilidad social se refiere a las condiciones y procesos relacionados con grupos susceptibles de sufrir daño o consecuencias negativas, un constructo complementario es la resiliencia (Bergstrand et al., 2015). Desde un enfoque psicológico se define como la capacidad de las personas para recuperarse de situaciones adversas y generadoras de estrés (Rutter, 1987), así como la capacidad para seguir haciendo frente a situaciones amenazantes o adversas (Bonnano, 2004).

La resiliencia abarca un grupo de indicadores o recursos como un estilo de afrontamiento activo, altos niveles de optimismo, sentido de propósito o la habilidad para gestionar emociones de una forma eficaz entre otros (Updegraff y Taylor, 2000; Zautra et al., 2010). Entendida de esta forma, la IE puede considerarse directamente conectada con la resiliencia, ya que las personas emocionalmente inteligentes saben adaptarse a situaciones adversas y amenazantes obteniendo resultados favorables o reduciendo los resultados negativos (Armstrong et al., 2011; Mikolajczak y Luminet, 2008; Salovey et al., 1999) o, cuanto menos, saben generar emociones y estados de ánimo que resultan beneficiosos a la hora de percibir y hacer frente a situaciones estresantes sobrevenidas (Keefer et al., 2009).

\section{Inteligencia emocional y violencia de género}

En las interacciones sociales, existen formas poco adaptativas de resolver los conflictos que tienen que ver con accio- 
nes destructivas y que se relacionan de forma negativa con el bienestar y con la satisfacción con las relaciones de pareja. Trabajos como el de Alonso-Ferres et al. (2019) muestran cómo niveles elevados de IE se relacionan con estilos de gestión de conflictos en la pareja de tipo constructivo dirigidos a la solución del problema y, en último término, con mayores niveles de bienestar y satisfacción con la relación de pareja. Igualmente, encuentran como la IE se relaciona negativamente con el empleo de formas de solución de conflictos más destructivas como querer mantener la defensa de los intereses propios mediante el control y el ejercicio de la oposición. Aunque estas relaciones se mantienen tanto en hombres como en mujeres, pueden resultar de especial utilidad en la violencia de género, actuando la IE como un factor protector de respuestas coercitivas y agresivas dirigidas hacia mujeres en situaciones de conflicto. En esta línea existen trabajos que confirman como niveles bajos de IE, concretamente una falta de empatía y de autorregulación emocional, se relacionan con tendencias agresivas en un grupo de hombres condenados por violencia de género (Jaffe et al., 2015); y otros trabajos encuentran que los hombres agresores condenados por violencia de género muestran niveles más bajos de IE que la población general y que, además, los déficits en algunas de las dimensiones de IE se relacionan con una mayor expresión de tendencias agresivas hacia sus parejas (Winters et al., 2004). Si el análisis de las habilidades emocionales se focaliza en las víctimas de violencia de género, los resultados parecen indicar que muestran déficits en estas habilidades cuando son comparadas con mujeres que no han sufrido violencia de género $\mathrm{y}$, además, muestran patrones de relaciones entre las dimensiones de la IE que no coinciden con los patrones del resto de mujeres (Tsirigotis y Luczak, 2016).

\section{Inteligencia emocional e inmigración}

La preocupación por el bienestar y la salud de la población inmigrante es una constante para los investigadores y las instituciones sociales. En muchas ocasiones los resultados dependen de los procesos de aculturación. En países como Alemania se ha comprobado como la reparación y la claridad emocional se relacionan de forma positiva con formas adaptativas de aculturación como la integración y negativamente con formas menos adaptativas como la separación o la marginalización (Schmitz y Schmitz, 2012). Igualmente, los autores encontraron cómo niveles elevados de IE en la población inmigrante se relacionan positivamente con un mejor ajuste psicológico y negativamente con la discriminación percibida. Cuando se analizan directamente las relaciones entre estrés percibido e IE en grupos de inmigrantes en España, se encuentra que una mayor atención a las emociones negativas y una menor reparación emocional se relacionan con mayores niveles de estrés (González-Castro et al., 2020).

\section{Inteligencia emocional y desempleo}

La IE se ha relacionado positivamente con los niveles de salud mental (menos depresión, ansiedad y estrés) y con la felicidad de un grupo de desempleados que buscaban de forma activa empleo (Peláez-Fernández et al., 2019). También la IE potencia los efectos de un rasgo de personalidad (core self-evaluations) sobre los niveles de felicidad subjetiva. Los beneficios de la IE, más allá de los resultados de estudios transversales, también se han demostrado con la aplicación de programas de intervención para la mejora de habilidades emocionales. Hodzic et al. (2015) muestran la eficacia de un programa para la mejora de habilidades emocionales en desempleados, pero solo en los casos que la situación de desempleo no se prolongaba en el tiempo. Las autoras comprueban los efectos positivos de su programa de intervención con un grupo de desempleados, seis meses después de su aplicación. Los desempleados que habían mejorado sus habilidades emocionales mostraban niveles más bajos de estrés, quejas somáticas y confusión, así como mejor salud mental y vigor. Las personas que pierden su empleo también podrían beneficiarse de los efectos positivos de la IE en lo que a las conductas de emprendimiento se refiere. Mortan et al. (2014), con una muestra de universitarios españoles y portugueses, muestran como la regulación emocional y el uso de emociones se relacionan positivamente con la intención de convertirse en una persona emprendedora a través de su efecto sobre los niveles de autoeficacia para el emprendimiento.

\section{Inteligencia emocional y fibromialgia}

En el sufrimiento de dolor crónico, todo parece indicar que los pacientes con fibromialgia constituyen un grupo especialmente vulnerable. Frente a otros pacientes con enfermedades reumáticas, las personas con fibromialgia cuentan con un menor nivel de recursos afectivos positivos, emplean menos estrategias efectivas de afrontamiento al dolor y tienen redes de apoyo social mucho más restringidas (Davis et al., 2001). La mayor parte de los estudios que relacionan los procesos emocionales con dolor incluyen muestras de pacientes con dolor generalizado sin diferenciar por grupos específicos. En una minoría se incluyen condiciones específicas como artritis reumatoide (Hamilton et al., 2005, 2007; Tillmann et al., 2013), dolor pélvico crónico (Thomas et al., 2006) o artritis reumatoide, esclerosis múltiple, espondilitis anquilosante y artritis reumatoide más una comorbilidad (Costa et al., 2014). Únicamente un estudio utiliza como muestra mujeres con fibromialgia (Van Middendorp et al., 2010) ya que, aunque en otros trabajos se identifican pacientes con fibromialgia, suponen un 10\% (Baker et al., 2016) y 14\% (González et al., 2007) de las muestras generales de dolor crónico, con tamaños muy reducidos y sin que en ningún momento los resultados sean analizados teniendo en cuenta el grupo de fibromial- 
gia. Además, el estudio de los procesos emocionales específicos o integrados bajo la etiqueta de IE se ha realizado con medidas de auto-informe o, empleando estas mismas medidas, partiendo de modelos rasgo/mixtos de IE, lo que en ningún caso permite analizar el rol de la IE como una habilidad en la percepción del dolor. Únicamente dos trabajos emplean medidas de habilidad, pero con pacientes con dolor crónico generalizado sin diferenciar pacientes con fibromialgia (Doherty et al., 2017; Zunhammer et al., 2015).

\section{Inteligencia emocional y mayores institucionalizados}

Todo parece indicar que con la edad, y fruto de la experiencia, la IE general y, específicamente, una habilidad como la regulación emocional, se incrementan en las personas (Kafetsios, 2004; Scheibe y Carstensen, 2009). No obstante, a partir de una edad elevada (por encima de los 70 años) se produce cierto deterioro en las habilidades emocionales (Cabello et al., 2014). Los estudios de la IE en la población de mayores son escasos y este déficit es más evidente cuando se contemplan grupos especialmente vulnerables como el de los mayores institucionalizados (Lloyd et al., 2012). La identificación de variables que promuevan y tengan un impacto positivo sobre el bienestar de este grupo es una prioridad (Almomani et al., 2014).

\section{Resultados y aportaciones del grupo TEAM ${ }^{+}$}

En el ámbito de la violencia de género, la importancia concedida a la cultura del honor se asocia con una mayor permisividad hacia prácticas violentas como una forma de restituir el honor, especialmente en el caso de los hombres. Los trabajos de nuestro grupo de investigación han mostrado cómo las personas con mayores niveles de IE otorgan menos importancia a la cultura del honor (incluyendo el honor masculino) lo que acaba redundando en una mayor satisfacción en la pareja y disminuyendo la probabilidad de que se produzcan conductas violentas en el seno de la pareja (Lopez-Zafra, Rodríguez-Espartal et al., 2008). Aunque no se llegan a encontrar diferencias en los niveles de IE en un estudio que compara un grupo de condenados por violencia de género, con un grupo de condenados por otros delitos y con un grupo de hombres que no han cometido delito alguno; sin embargo, en el primer grupo sí encontramos una relación positiva entre los niveles de atención emocional y uno de los factores de la cultura del honor (honor individual) (Torres Fúnez y Lopez-Zafra, 2010). Una de las aportaciones más relevantes del grupo es la creación y aplicación de un programa de intervención de tipo emocional dirigido a presos condenados por violencia de género (Rodríguez-Espartal y Lopez-Zafra, 2013). Cuando comparamos los resultados obtenidos tras la aplicación del programa emocional con los que resultan de la aplicación de un programa cognitivo-conductual y un grupo control, encontramos que se produce una reducción en los pensamientos distorsionados sobre la mujer y sobre el uso de la violencia, obteniendo los mejores resultados en el grupo en el que se ha aplicado el programa de tipo emocional.

Profundizando en los estudios que relacionan la IE con los procesos de aculturación en población inmigrante, nuestro grupo estudia en un grupo de mujeres inmigrantes en España de origen marroquí ese tipo de relaciones, así como la influencia de variables sociales como la cultura del honor (Lopez-Zafra y El Ghoudani, 2014). La IE se relaciona positivamente con las estrategias de integración y negativamente con las de asimilación. Además, la IE, cumpliendo una función adaptativa, media en la relación entre la cultura del honor y las estrategias de integración ayudando a crear un equilibrio entre la cultura de origen y la cultura de destino.

Con base en los estudios que plantean cómo los procesos auto-regulatorios pueden proteger la salud mental de las personas que pierden su empleo, nuestro trabajo (Berrios et al., 2016) analiza las relaciones que la IE mantiene en un grupo de 442 empleados con distintas medidas de ajuste psicológico. Igualmente, comprueba si la IE consigue explicar un porcentaje de varianza de los niveles de ajuste psicológico, más allá de lo explicado por recursos como el apoyo social. Encontramos relaciones negativas entre los niveles de IE y síntomas depresivos y estrés; y relaciones positivas entre IE y la satisfacción con la vida y felicidad. Además, una habilidad específica, el uso de emociones, predice los niveles de depresión y bienestar subjetivo, más allá de lo explicado por el apoyo social. También hemos analizado el papel que la IE juega en el afrontamiento al estrés generado por la pérdida del empleo y cómo puede actuar como una variable potenciadora del uso de estrategias de empleo activas (Nieto-Flores et al., 2019), encontrando que las habilidades emocionales incrementan la autopercepción de eficacia en la búsqueda activa de empleo lo que, en último término, determina las conductas de búsqueda activa de empleo en desempleados.

Nuestros estudios con pacientes que sufren enfermedades crónicas, concretamente fibromialgia, han mostrado la contribución que una habilidad de la IE como es la regulación de las propias emociones tiene sobre los niveles de resiliencia experimentados por estos pacientes. La regulación emocional, junto a los niveles de optimismo y afecto positivo experimentado, consiguen establecer diferencias entre dos perfiles de los pacientes con fibromialgia que son determinantes en el establecimiento de diferencias en la severidad de la enfermedad (perfil de adaptación y de mala adaptación). Así las personas con mayores niveles de regulación emocional sabrán adaptarse de una forma eficaz a la enfermedad logrando un menor nivel de severidad (EstévezLópez et al., 2017). Mediante la aplicación de técnicas 
SEM, hemos conseguido demostrar la existencia de una vía de mecanismos psicológicos, caracterizada por altos niveles de resiliencia (comprendiendo entre otros, altos niveles de regulación emocional) y bajos niveles de catastrofismo que pueden reducir la severidad de la fibromialgia experimentada por los pacientes (Pulido-Martos et al., 2020). Centrándonos en que la fibromialgia en el $90 \%$ de los casos es diagnosticada en mujeres, tratamos de buscar diferencias en los niveles de IE como habilidad en un grupo de mujeres diagnosticadas de fibromialgia y un grupo de mujeres sanas, así como la relación que la IE mantiene con el dolor generalizado percibido en mujeres con fibromialgia (Luque -Reca et al., 2019). Nuestros resultados muestran que la comprensión emocional se encuentra más deteriorada en mujeres con fibromialgia y que la percepción y regulación emocional son capaces de explicar los niveles de dolor generalizado, aun cuando se controlan los niveles de catastrofismo.

Con relación al grupo de mayores institucionalizados, nuestros trabajos analizan el papel que la IE juega en la explicación de la calidad de vida experimentada (Luque-Reca et al., 2018) y los niveles de sintomatología depresiva (Luque -Reca et al., 2016). En el primer caso hemos podido comprobar cómo la IEP ejerce su efecto sobre diferentes dimensiones de la calidad de vida subjetiva de los mayores a través de los estilos cognitivos optimista y pesimista. Concretamente el estilo optimista media entre los niveles de IEP y salud; mientras que el pesimismo media entre la IEP y una realización reducida de actividades en el tiempo libre (Luque-Reca et al., 2018). En la misma línea, tratando de analizar el impacto que la IE tiene en los niveles de bienestar de nuestros mayores institucionalizados y considerando los efectos beneficiosos de la práctica de actividad física, hemos comprobado que habilidades emocionales como la percepción de emociones ajenas y el uso de emociones se asocian de forma positiva con la práctica de actividad física en este grupo, aun cuando se controla la influencia de rasgos de personalidad (Pulido-Martos et al., 2014).

\section{Conclusiones}

De todos los puntos tratados y de nuestras contribuciones a su estudio, podemos destacar algunas cuestiones. En primer lugar, hemos podido comprobar que la IE juega un papel relevante en procesos psicosociales que tienen que ver con las relaciones interpersonales en distintos contextos (hospitalario, educativo, laboral...). Sin embargo, para comprender el papel de este constructo es necesario atender a la realidad de cada colectivo, así como al modelo de partida de la IE y el rol específico de las dimensiones que la forman. De la misma forma, para profundizar en las relaciones entre las variables objeto de estudio, es necesario tener en cuenta diferentes niveles de análisis (nivel individual, grupal, organizacional...). En general, nuestros resul- tados son coherentes con los de otras investigaciones llevadas a cabo en diferentes países, y confirman la hipótesis de que la IE actúa como un recurso personal que amortigua los efectos negativos del estrés y del burnout en colectivos con especial incidencia, como el de enfermería y profesorado. Asimismo, la IE mejora diferentes indicadores del bienestar subjetivo de estos profesionales y de trabajadores de otros contextos laborales. Es decir, por un lado, la IE se muestra como un factor facilitador de resultados saludables: satisfacción laboral, satisfacción vital, bienestar psicológico, mejores relaciones sociales y/o de pareja, mejor rendimiento; y por otro, como un factor protector de diferentes riesgos: estrés, burnout, dolor en la enfermedad, relaciones de pareja violentas... Estos efectos no solo ocurren a nivel individual, sino que también afectan a los procesos grupales como el ejercicio del liderazgo en diferentes contextos. Por ejemplo, los líderes o directivos de las empresas, de un centro de salud u hospitalario o de un aula. Así, se ha comprobado que si los profesores están menos estresados y más satisfechos con su vida podrán llevar a cabo prácticas óptimas de enseñanza que contribuyan al mejor funcionamiento del aula y al éxito escolar. Igualmente, los resultados a nivel grupal en el liderazgo implican que, mejorando las habilidades emocionales del líder, se pueden mejorar las habilidades grupales, por lo que una mejora de la IE del líder puede producir también mejoras en el liderazgo ejercido y la satisfacción global de los trabajadores.

Todo ello nos lleva a concluir que es necesario intervenir en distintos contextos de trabajo y/o sociales. De hecho, se ha comprobado meta-analíticamente, que el entrenamiento en IE tiene un efecto moderado y positivo y mejora los resultados en el ámbito laboral por lo que puede considerarse una "buena inversión" (Mattingly y Kraiger, 2019). Esto permitiría dotar a los individuos de recursos personales que les permitan afrontar adecuadamente las demandas laborales o académicas, liderar mejor y tener mejores relaciones interpersonales. De acuerdo con Salanova et al. (2003), sería necesario realizar acciones informativas y divulgativas para concienciar de la importancia de estas habilidades, llevar a cabo acciones de formación continua sobre temas específicos (p.ej., burnout o estrategias de motivación), así como la realización de talleres de trabajo eminentemente prácticos y adaptados a los colectivos y/o a centros específicos (workshops) para entrenar las habilidades de la IE (percepción, expresión, facilitación, comprensión y regulación emocional). En cuanto a los programas de intervención existente, los datos son heterogéneos. Así, en el ámbito de la enfermería la mayor parte de los programas se han realizado en el contexto del liderazgo y el desarrollo organizacional, centrados en personas y equipos, y solamente en uno de los casos se informa de la eficacia del programa con enfermeras de oncología (Codier et al., 2013). En el ámbito educativo existe un mayor número de expe- 
riencias centradas en el entrenamiento de las habilidades socio-emocionales que promueven el bienestar y el rendimiento de los estudiantes y los docentes. Aunque en la mayoría de los casos se llevan a cabo iniciativas ocasionales no sistematizadas en las que se realizan reflexiones personales de forma aleatoria, discontinua y a veces contradictoria que no suele evaluarse de manera rigurosa. Esto contrasta, por una parte, con el interés y motivación creciente de los profesores por dotar al alumnado no solo de conocimientos, sino también de habilidades socio-emocionales; y por otra, con los resultados de diferentes estudios empíricos que han demostrado que la formación organizada y bien estructurada es muy útil para promover conductas saludables en los alumnos (Weissberg y O'Brien, 2004). Por tanto, además de extender el uso de intervenciones ya existentes con alumnado (p.ej., INTEMO o RULER), se debería profundizar en la mejora y evaluación de los mismos. Esto también sucede en otros contextos como el de la violencia de género, en el que la aplicación de un programa como PREMOVIGE (Programa emocional para presos por violencia de género) contribuye a la reducción de los pensamientos distorsionados que los condenados por este delito tienen sobre la mujer y el uso de la violencia. Esto ayuda a normalizar las relaciones que los presos, en algunos casos, siguen manteniendo con las víctimas una vez que cumplen su condena. Los beneficios de estos programas en hombres agresores también se han encontrado en muestras de adolescentes (Garaigordobil y Peña-Sarrionandia, 2015), por lo que es importante seguir incidiendo en estos procesos.

En otros grupos vulnerables, la mejora de habilidades emocionales también favorece procesos como la aculturación entre la población inmigrante, lo que ayudaría a detectar los grupos con mayores problemas de adaptación y mejorarla. En el caso de personas desempleadas el desarrollo de habilidades socio-emocionales a través de los programas de orientación vocacional aplicados en las etapas de formación de nuestros jóvenes, potenciará el bienestar y mejoraría la implicación en una búsqueda activa de empleo. Con relación a los pacientes crónicos, en el caso de diagnósticos de fibromialgia, es de especial importancia determinar el perfil de ajuste de estos pacientes, y en los casos de perfiles de mala adaptación, incrementar los niveles de resiliencia (incluyendo sus habilidades de regulación emocional) llevaría a una menor severidad de la enfermedad, lo que contribuye a experimentar niveles más bajos de dolor generalizado, que es uno de los síntomas recurrentes en las distintas propuestas de criterios diagnósticos de la enfermedad. Finalmente, en los mayores institucionalizados, estas intervenciones pueden mejorar sus niveles de calidad de vida y disminuir el riesgo de padecer síntomas depresivos, beneficiándose de programas dirigidos a la mejora de los niveles de IE y de la auto-eficacia emocional. Además, eso puede contribuir a una mayor realización de actividad física en esta población, con todos los beneficios asociados que esa práctica conlleva.

En resumen, sigue siendo de interés profundizar en el papel que la IE tiene en distintos procesos y diferentes colectivos. Por ello, nuestro grupo propone seguir avanzando en el análisis de los procesos de mediación y/o moderación de las relaciones de la IE con diferentes variables de tipo psicosocial así como mejorar los resultados en estas variables a través de la intervención en IE. Es decir, mejorando la IE del grupo, así como la IE individual, e interviniendo en distintos niveles, podremos seguir comprendiendo este proceso y mejorando el bienestar y la salud psicosocial. De ahí, que nuestro equipo siga trabajando precisamente en estas temáticas, incidiendo especialmente en las líneas sobre las que se debe profundizar (p.ej., análisis multinivel, intervención o estudios longitudinales). Con estas contribuciones, podemos seguir aportando al excelente trabajo que se realiza desde nuestro país y que contribuye al avance del conocimiento en la IE y su aplicación.

La Figura 1 recoge un resumen de todas las aportaciones del grupo TEAM ${ }^{+}$. 


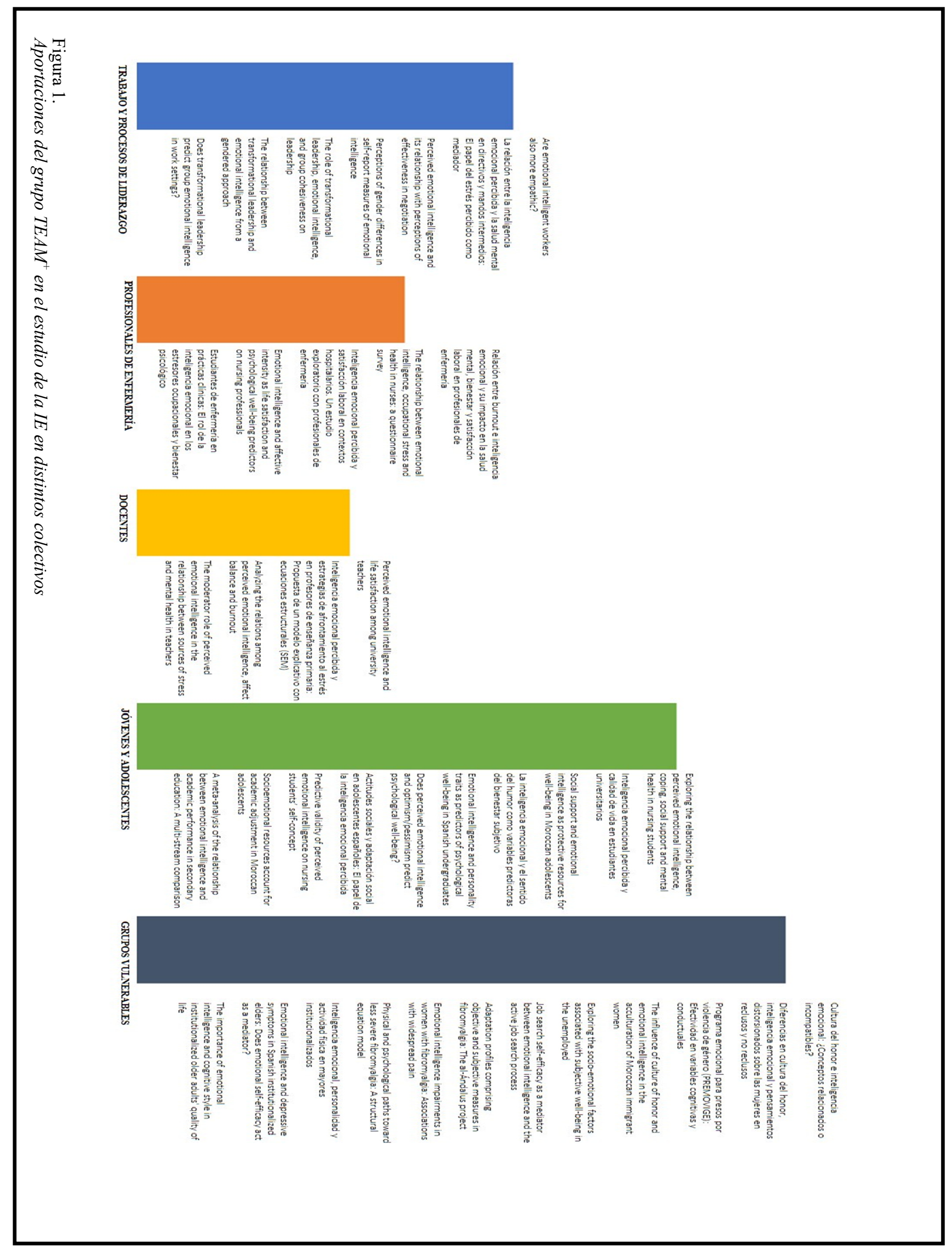




\section{Referencias}

Almomani, F.M., Mcdowd, J.M., Bani-Issa, W. y Almomani, M. (2014). Health-related quality of life and physical, mental, and cognitive disabilities among nursing home residents in Jordan. Quality of Life Research, 23, 155-165. https://doi.org/10.1007/s11136 $-013-0461-2$

Alonso-Ferres, M., Valor-Segura, I. y Expósito, F. (2019). Couple conflict-facing responses from a gender perspective: Emotional intelligence as a differential pattern. Psychosocial Intervention, 28, 147-156. https://doi.org/10.5093/pi2019a9

Armstrong, A.R., Galligan, R.F. y Critchley, C.R. (2011). Emotional intelligence and psychological resilience to negative life events. Personality and Individual Differences, 51(3), 331-336. https://doi.org/10.1016/ j.paid.2011.03.025

Ashkanasy, N.M. y Dorris, A.D. (2017). Emotions in the workplace. Annual Review of Organizational Psychology and Organizational Behaviour, 4, 6790. https://doi.org/10.1146/annurev-orgpsych032516-113231

Augusto-Landa, J.M., Berrios, M.P., Lopez-Zafra, E. y Aguilar-Luzón, M.C. (2006). Relación entre burnout e inteligencia emocional y su impacto en la salud mental, bienestar y satisfacción laboral en profesionales de enfermería. Ansiedad y Estrés, 12(2-3), 479 -493 .

Augusto-Landa, J.M., Lopez-Zafra, E., Aguilar-Luzón, M.C. y Salguero, M.F. (2009). Predictive validity of perceived emotional intelligence on nursing students' self-concept. Nurse Education Today, 29(7), 801 -808. https://doi.org/10.1016/j.nedt.2009.04.004

Augusto-Landa, J.M., Lopez-Zafra, E., Berrios, M.P. y Aguilar-Luzón, M.C. (2008). The relationship between emotional intelligence, occupational stress and health in nurses: a questionnaire survey. International Journal of Nursing Studies, 45(6), 888-901. https://doi.org/10.1016/j.ijnurstu.2007.03.005

Augusto-Landa, J.M., Lopez-Zafra, E., Berrios, M.P. y Pulido-Martos, M. (2012). Analyzing the relations among perceived emotional intelligence, affect balance and burnout. Behavioral Psychology / Psicología Conductual, 20(1), 151-168.

Augusto-Landa, J.M., Lopez-Zafra, E., Martínez de Antoñana, R. y Pulido-Martos, M. (2006). Perceived emotional intelligence and life satisfaction among university teachers. Psicothema, 18 Sup., 152-157.

Augusto-Landa, J.M., Lopez-Zafra, E. y Pulido-Martos, M.
(2011). Inteligencia emocional percibida y estrategias de afrontamiento al estrés en profesores de enseñanza primaria: propuesta de un modelo explicativo con ecuaciones estructurales (SEM). Revista de Psicología Social, 26(3), 413-425. https:// doi.org/10.1174/021347411797361310

Augusto-Landa, J.M., Martínez, R., Pulido-Martos, M., Berrios, M.P., Lopez-Zafra, E. y Luque, P. (2004). Inteligencia emocional percibida y calidad de vida en estudiantes universitarios. Revista de Psicología Social Aplicada, 14(2), 61-78.

Augusto-Landa, J.M., Pulido-Martos, M. y Lopez-Zafra, E. (2010). Emotional intelligence and personality traits as predictors of psychological well-being in Spanish undergraduates. Social Behavior and Personality: An International Journal, 38(6), 783-793. https:// doi.org/10.2224/sbp.2010.38.6.783

Augusto-Landa, J.M., Pulido-Martos, M. y Lopez-Zafra, E. (2011). Does perceived emotional intelligence and optimism/pessimism predict psychological wellbeing? Journal of Happiness Studies, 12(3), 463474. https://doi.org/10.1007/s10902-010-9209-7

Ayiro, L.P. (2014). Transformational leadership and ochool outcomes in Kenya: Does emotional intelligence matter? FIRE - Forum for International Research in Education, 1(1), 26-49. https://doi.org/10.18275/ fire201401011011

Baker, K.S., Gibson, S., Georgiou-Karistianis, N., Roth, R.M. y Giummarra, M.J. (2016). Everyday executive functioning in chronic pain: Specific deficits in working memory and emotion control, predicted by mood, medications, and pain interference. Clinical Journal of Pain, 32(8), 673-680. https:// doi.org/10.1097/AJP.0000000000000313

Bergstrand, K., Mayer, B., Brumback, B. y Zhang, Y. (2015). Assessing the relationship between social vulnerability and community resilience to hazards. Social Indicators Research, 122(2), 391-409. https:// doi.org/10.1007/s11205-014-0698-3

Berrios, M.P., Augusto-Landa, J.M. y Aguilar Luzón, M.C. (2006). Inteligencia emocional percibida y satisfacción laboral en contextos hospitalarios. Un estudio exploratorio con profesionales de enfermería. Index de Enfermería, 15(54), 30-34.

Berrios, M.P., Extremera, N. y Nieto-Flores, M.P. (2016). Exploring the socio-emotional factors associated with subjective well-being in the unemployed. PeerJ, 4, e2506. https://doi.org/10.7717/peerj.2506

Berrios, M.P., Lopez-Zafra, E., Pulido-Martos, M. y Augusto-Landa, J.M. (2013). Are emotional intelligent 
workers also more empathic? Scandinavian Journal of Psychology, 54(5), 407-414. https:// doi.org/10.1111/sjop.12058

Berrios, M.P., Martos-Luque, R. y Martos-Montes, R. (2016). Crecer con emoción: Una experiencia educativa en el aula de emociones aplicando el programa "Emo-é". Padres y Maestros, 368, 36-42. https://doi.org/10.14422/pym.i368.y2016.006

Berrios, M.P., Pulido-Martos, M., Augusto-Landa, J.M. y Lopez-Zafra, E. (2012). La inteligencia emocional y el sentido del humor como variables predictoras del bienestar subjetivo. Behavioral Psychology / Psicología Conductual, 20(1), 211-227.

Blanco, G. (2004). Estrés laboral y salud en las enfermeras instrumentistas. Revista de la Facultad de Medicina, 27(1), 29-35.

Bonanno, G.A. (2004). Loss, trauma, and human resilience: have we underestimated the human capacity to thrive after extremely aversive events? American Psychologist, 59(1), 20-28. https:// doi.org/10.1037/0003-066X.59.1.20

Brackett, M.A., Palomera, R., Mojsa-Kaja, J., Reyes, M.R. y Salovey, P. (2010). Emotion-regulation ability, burnout, and job satisfaction among British secondary-school teachers. Psychology in the Schools, 47 (4), 406-417. https://doi.org/10.1002/pits.20478

Brackett, M.A., Rivers, S.E., Reyes, M.R. y Salovey, P. (2012). Enhancing academic performance and social and emotional competence with the RULER feeling words curriculum. Learning and Individual Differences, 22(2), 218-224. http://dx.doi.org/10.1016/ j.lindif.2010.10.002

Braun, S., Peus, C., Weisweiler, S. y Frey, D. (2013). Transformational leadership, job satisfaction, and team performance: A multilevel mediation model of trust. The Leadership Quarterly, 24(1), 270283. https://doi.org/10.1016/j.leaqua.2012.11.006

Burton, C., Rufat, S. y Tate, E. (2018). Social Vulnerability. En S. Fuchs y T. Thaler (Eds.), Vulnerability and Resilience to Natural Hazards (pp. 53-81). Cambridge University Press.

Cabello, R., Navarro, B., Latorre, J.M. y FernándezBerrocal, P. (2014). Ability of university level education to prevent age-related decline in emotional intelligence. Frontiers in Aging Neuroscience, 6 (37), 1-7. https://doi.org/10.3389/fnagi.2014.00037

Cabello, R., Ruiz-Aranda, D. y Fernández-Berrocal, P. (2010). Docentes emocionalmente inteligentes. Revista Electrónica Interuniversitaria de Formación del Profesorado, 13 (1), 41-49.

Carson, R.L., Plemmons, S., Templin, T.J. y Weiss, H.M. (2011). "You are who you are:" A mixed-method study of affectivity and emotion regulation in curbing teacher burnout. En G. M. Reevy y E. Frydenberg (Eds.), Personality, Stress, and Coping: Implications for Education (pp. 239-265). IAP Information Age Publishing.

Cherniss, C. y Roche, C.W. (2020). Leading With Feeling: Nine Strategies of Emotionally Intelligent Leadership. Oxford University Press.

Clouder, C., Dahlin, B., Diekstra, R., Fernández-Berrocal, P., Heys, B., Lantieri, L. y Paschen, H. (2008). Educación Emocional y Social. Análisis Internacional. Informe Fundación Marcelino Botín 2008. Fundación Botín.

Codier, E., Freitas, B. y Muneno, L. (2013). Developing emotional intelligence ability in oncology nurses: A clinical rounds approach. Oncology Nursing Forum, 40(1), 22-29. https://doi.org/10.1188/13.ONF.22-29

Cortés-Denia, D., El Ghoudani, K., Pulido-Martos, M., Alaoui, S., Luque-Reca, O., Ramos-Álvarez, M.M., Augusto-Landa, J.M., Zarhbouch, B. y Lopez-Zafra, E. (2020). Socioemotional resources account for academic adjustment in Moroccan adolescents. Frontiers in Psychology, 11, 1609. https:// doi.org/10.3389/fpsyg.2020.01609

Costa, S., Petrides, K.V. y Tillmann, T. (2014). Trait emotional intelligence and inflammatory diseases. Psychology, Health \& Medicine, 19(2), 180-189. https://doi.org/10.1080/13548506.2013.802356

Cummings, G., Hayduk, L. y Estabrooks, C. (2005). Mitigating the impact of hospital restructuring on nurses: The responsibility of emotionally intelligent leadership. Nursing Research, 54(1), 2-12. https:// doi.org/10.1097/00006199-200501000-00002

Cutter, S.L., Boruff, B.J. y Shirley, W.L. (2003). Social vulnerability to environmental hazards. Social Science Quarterly, 84(1), 242-261. https:// doi.org/10.1111/1540-6237.8402002

Davis, M.C., Zautra, A.J. y Reich, J.W. (2001). Vulnerability to stress among women in chronic pain from fibromyalgia and osteoarthritis. Annals of Behavioral Medicine, 23(3), 215-226. https:// doi.org/10.1207/S15324796ABM2303_9

Doherty, E.M., Walsh, R., Andrews, L. y McPherson, S. (2017). Measuring emotional intelligence enhances the psychological evaluation of chronic pain. Jour- 
nal of Clinical Psychology in Medical Settings, 24(3 -4), 365-375. https://doi.org/10.1007/s10880-0179515-x

Estévez-López, F., Segura-Jiménez, V., Álvarez-Gallardo, I.C., Borges-Cosic, M., Pulido-Martos, M., Carbonell-Baeza, A., Aparicio, V.A., Geenen, R. y Delgado-Fernández, M. (2017). Adaptation profiles comprising objective and subjective measures in fibromyalgia: The al-Ándalus project. Rheumatology, 56(11), 2015-2024. https://doi.org/10.1093/ rheumatology/kex302

Extremera, N. y Fernández-Berrocal, P. (2003). La inteligencia emocional en el contexto educativo: Hallazgos científicos de sus efectos en el aula. Revista de Educación, 332, 97-116.

Extremera, N., Fernández-Berrocal, P. y Durán, A. (2003). Inteligencia emocional y burnout en profesores. Encuentros en Psicología Social, 1(5), 260-265.

Fernández-Berrocal, P. y Extremera, N. (2005). La inteligencia emocional y la educación de las emociones desde el modelo de Mayer y Salovey. Revista Interuniversitaria de Formación del Profesorado, 19(3), 63-93.

Fernández-Berrocal, P. y Extremera, N. (2010). Más Aristóteles y menos Prozac: La inteligencia emocional y el estudio de la felicidad. Encuentros en Psicología Social, 5(1), 40-51.

Garaigordobil, M. y Peña-Sarrionandia, A. (2015). Effects of an emotional intelligence program in variables related to the prevention of violence. Frontiers in Psychology, 6, 743. https://doi.org/10.3389/ fpsyg.2015.00743

González, V., Ramírez-Maestre, C. y Herrero, A.M. (2007). Inteligencia emocional, personalidad y afrontamiento en pacientes con dolor crónico. Revista Mexicana de Psicología, 24(2),185-195.

González-Castro, J.L., Ubillos Landa, S., Puente Martínez, A. y Vera Perea, M. (2020). The role of emotional intelligence and sociocultural adjustment on migrants' self-reported mental well-being in Spain: A 14 month follow-up study. International Journal of Environmental Research and Public Health, 17(4), 1206. https://doi.org/10.3390/ijerph17041206

Görgens-Ekermans, G. y Brand, T. (2012). Emotional intelligence as a moderator in the stress-burnout relationship: A questionnaire study on nurses. Journal of Clinical Nursing, 21(15-16), 2275-2285. https:// doi.org/10.1111/j.1365-2702.2012.04171.x

Greenberg, M.T., Weissberg, R.P., O’brien, M.U., Zins,
J.E., Fredericks, L., Resnik, H. y Elias, M.J. (2003). Enhancing school-based prevention and youth development through coordinated social, emotional, and academic learning. American Psychologist, 58(6-7), 466-474. https://doi.org/10.1037/0003-066X.58.67.466

Hagelskamp, C., Brackett, M.A., Rivers, S.E. y Salovey, P. (2013). Improving classroom quality with the ruler approach to social and emotional learning: Proximal and distal outcomes. American Journal of Community Psychology, 51(3-4), 530-543. http:// dx.doi.org/10.1007/s10464-013-9570-x

Hamilton, N.A., Zautra, A.J. y Reich, J.W. (2005). Affect and pain in rheumatoid arthritis: Do individual differences in affective regulation and affective intensity predict emotional recovery from pain? Annals of Behavioral Medicine, 29(3), 216-224. https:// doi.org/10.1207/s15324796abm2903_8

Hamilton, N.A., Zautra, A.J. y Reich, J. (2007). Individual differences in emotional processing and reactivity to pain among older women with rheumatoid arthritis. Clinical Journal of Pain, 23(2), 165-172. https:// doi.org/10.1097/AJP.0b013e31802b4f58

Hargreaves, A. (2000). Mixed emotions: Teachers' perceptions of their interactions with students. Teaching and Teacher Education, 16(8), 811-826. http:// doi.org/10.1016/S0742-051X(00)00028-7

Hodzic, S., Ripoll, P., Bernal, C. y Zenasni, F. (2015). The effects of emotional competences training among unemployed adults: A longitudinal study. Applied Psychology: Health and Well-Being, 7(3), 275-292. https://doi.org/10.1111/aphw.12048

Hong, E. y Lee, Y. S. (2016). The mediating effect of emotional intelligence between emotional labour, job stress, burnout and nurses' turnover intention. International Journal of Nursing Practice, 22(6), 625632. https://doi.org/10.1111/ijn.12493

Jaffe, A.E., Simonet, D.V., Tett, R.P., Swopes, R.M. y Davis, J.L. (2015). Multidimensional trait emotional intelligence and aggressive tendencies in male offenders of domestic violence. Journal of Family Violence, 30(6), 769-781. https://doi.org/10.1007/ s10896-015-9729-3

Jiménez, M.I. y Lopez-Zafra, E. (2011). Actitudes sociales y adaptación social en adolescentes españoles: el papel de la inteligencia emocional percibida. Revista de Psicología Social, 26(1), 105-117. https:// doi.org/10.1174/021347411794078417

Kafetsios, K. (2004). Attachment and emotional intelligen- 
ce abilities across the life course. Personality and Individual Differences, 37(1), 129-145. https:// doi.org/10.1016/j.paid.2003.08.006

Keefer, K.V., Parker, J.D.A. y Saklofske, D.H. (2009). Emotional Intelligence and Physical Health. En C. Stough, D.H. Saklofske y J.D.A. Parker (Eds.), $A s$ sessing Emotional Intelligence: Theory, Research, and Applications (pp. 191-218). Springer.

Kramer, M., Page, L. y Klemic, G. (2019). Evolving leadership: New clues and cues toward environment and context. Journal of Leadership Studies, 12(4), 82-85. https://doi.org/10.1002/j1s.21618

Li, H., Cheng, B. y Zhu, X.P. (2018). Quantification of burnout in emergency nurses: A systematic review and meta-analysis. International Emergency Nursing, 39, 46-54. https://doi.org/10.1016/ j.ienj.2017.12.005

Lloyd, S.J., Malek-Ahmadi, M., Barclay, K., Fernandez, M.R. y Chartrand, M.S. (2012). Emotional intelligence (EI) as a predictor of depression status in older adults. Archives of Gerontology and Geriatrics, 55(3), 570-573. https://doi.org/10.1016/ j.archger.2012.06.004

Lopes, P.N. (2016). Emotional intelligence in organizations: Bridging research and practice. Emotion Review, $\quad 8(4), \quad 316-321 . \quad \mathrm{https}: / /$ doi.org/10.1177/1754073916650496

Lopez-Zafra, E., y El Ghoudani, K. (2014). The influence of culture of honor and emotional intelligence in the acculturation of Moroccan immigrant women. The Spanish Journal of Psychology, 17, E49. https:// doi.org/10.1017/sjp.2014.53

Lopez-Zafra, E., Garcia-Retamero, R. y Augusto-Landa, J.M. (2008). The role of transformational leadership, emotional intelligence, and group cohesiveness on leadership. Journal of Leadership Studies, 2, 37-49. https://doi.org/10.1002/j1s.20074

Lopez-Zafra, E., Garcia-Retamero, R. y Berrios, M.P. (2012). The relationship between transformational leadership and emotional intelligence from a gendered approach. Psychological Record, 62, 97-114. https://doi.org/10.1007/BF03395790

Lopez-Zafra E. y Gartzia, L. (2014). Perceptions of gender differences in self-report measures of emotional intelligence. Sex Roles: A Journal of Research, 70 (11-12), 479-495. https://doi.org/10.1007/s11199014-0368-6

Lopez-Zafra, E. y Pulido-Martos, M. (2020). Inteligencia emocional en las organizaciones. Síntesis.
Lopez-Zafra, E., Pulido-Martos, M., Berrios, M.P. y Augusto-Landa, J.M. (2017). Does transformational leadership predict group emotional intelligence in work settings? Revista de Psicología Social, 32(3), 513-538. https:// doi.org/10.1080/02134748.2017.1352170

Lopez-Zafra, E., Ramos-Álvarez, M.M., El Ghoudani, K., Luque-Reca, O., Augusto-Landa, J.M., Zarhbouch, B., Alaoui, S., Cortés-Denia, D. y Pulido-Martos, M. (2019). Social support and emotional intelligence as protective resources for well-being in Moroccan adolescents. Frontiers in Psychology, 10, 1529. https://doi.org/10.3389/fpsyg.2019.01529.

Lopez-Zafra, E., Rodríguez-Espartal, N. y Jiménez Morales, M.I. (2008). Cultura del honor e inteligencia emocional: ¿Conceptos relacionados o incompatibles? Summa Psicológica UST, 5(2), 17-26. https:// doi.org/10.18774/448x.2008.5.105

Lord, R.G., Day, D.V., Zaccaro, S.J., Avolio, B.J. y Eagly, A.H. (2017). Leadership in applied psychology: Three waves of theory and research. Journal of Applied Psychology, 102(3), 434-451. https:// doi.org/10.1037/ap10000089

Loughhead, S. y Mittai, O. (2000). Urban poverty and vulnerability in India: A social perspective [Comunicación oral]. Urban Forum: Urban Poverty Reduction in the 21st Century, Chantilly, Virginia.

Luque-Reca, O., Augusto-Landa, J.M. y Pulido-Martos, M. (2014). La relación entre la inteligencia emocional percibida y la salud mental en directivos y mandos intermedios: El papel del estrés percibido como mediador. Ansiedad y Estrés, 20(1), 61-73.

Luque-Reca, O., Augusto-Landa, J.M. y Pulido-Martos, M. (2016). Emotional intelligence and depressive symptoms in Spanish institutionalized elders: Does emotional self-efficacy act as a mediator? PeerJ, 4, e2246. https://doi.org/10.7717/peerj.2246

Luque-Reca, O., Pulido-Martos, M., Gavilán-Carrera, B., García-Rodríguez, I.C., McVeigh, J.G., Aparicio, V.A. y Estévez-López, F. (2019). Emotional intelligence impairments in women with fibromyalgia: Associations with widespread pain. Journal of Health Psychology. Publicación avanzada en línea. https://doi.org/10.1177/1359105319890916

Luque-Reca, O., Pulido-Martos, M., Lopez-Zafra, E. y Augusto-Landa, J.M. (2018). The importance of emotional intelligence and cognitive style in institutionalized older adults' quality of life. Journal of General Psychology, 145(2), 120-133. https:// doi.org/10.1080/00221309.2018.1437384 
Mayer, J.D., Roberts, R.D. y Barsade, S.G. (2008). Human abilities: Emotional intelligence. Annual Review Psychology, 59, 507-536. https://doi.org/10.1146/ annurev.psych.59.103006.093646

MacCann, C., Joseph, D.L., Newman, D.A., y Roberts, R.D. (2014). Emotional intelligence is a secondstratum factor of intelligence: Evidence from hierarchical and bifactor models. Emotion, 14, 358-374. http://dx.doi.org/10.1037/a0034755

Mattingly, V. y Kraiger, K. (2019). Can emotional intelligence be trained? A meta-analytical investigation. Human Resource Management Review, 29(2), 140155. https://doi.org/10.1016/j.hrmr.2018.03.002

McTiernan, K. y McDonald, N. (2015). Occupational stressors, burnout and coping strategies between hospital and community psychiatric nurses in a Dublin region. Journal of Psychiatric and Mental Health Nursing, 22(3), 208-218. https://doi.org/10.1111/ jpm. 12170

Miao, C., Humphrey, R.H. y Qian, S. (2016). Leader emotional intelligence and subordinate job satisfaction: A meta-analysis of main, mediator, and moderator effects. Personality and Individual Differences, 102, 13-24. http://dx.doi.org/10.1016/j.paid.2016.06.056

Mikolajczak, M. y Luminet, O. (2008). Trait emotional intelligence and the cognitive appraisal of stressful events: An exploratory study. Personality and Individual Differences, 44(7), 1445-1453. https:// doi.org/10.1016/j.paid.2007.12.012

Montes-Berges, B. y Augusto-Landa, J.M. (2007). Exploring the relationship between perceived emotional intelligence, coping, social support and mental health in nursing students. Journal of Psychiatric and Mental Health Nursing, 14(2), 163-171. https:// doi.org/10.1111/j.1365-2850.2007.01059.x

Montes-Berges, B. y Augusto-Landa, J.M. (2014). Emotional intelligence and affective intensity as life satisfaction and psychological well-being predictors on nursing professionals. Journal of Professional Nursing, 30(1), 80-88. https://doi.org/10.1016/ j.profnurs.2012.12.012

Morese, R., Palermo, S., Defedele, M., Nervo, J. y Borraccino, A. (2019). Vulnerability and Social Exclusion: Risk in Adolescence and Old Age. En R. Morese y S. Palermo (Eds.), The New Forms of Social Exclusion. IntechOpen.

Moriana, J.A. y Herruzo, J. (2004). Estrés y burnout en profesores. International Journal of Clinical and Health Psychology, 4(3), 597-621.
Mortan, R.A., Ripoll, P., Carvalho, C. y Bernal, M.C. (2014). Effects of emotional intelligence on entrepreneurial intention and self-efficacy. Journal of Work and Organizational Psychology, 30, 97-104. http://dx.doi.org/10.1016/j.rpto.2014.11.004

Nieto-Flores, M.P., Berrios, M.P. y Extremera, N. (2019). Job search self-efficacy as a mediator between emotional intelligence and the active job search process. International Journal of Social Psychology, 34(1), 86-109. doi.org/10.1080/02134748.2018.1537652

Peláez-Fernández, M.A., Rey, L. y Extremera, N. (2019). Psychological distress among the unemployed: Do core self-evaluations and emotional intelligence help to minimize the psychological costs of unemployment? Journal of Affective Disorders, 256, 627632. https://doi.org/10.1016/j.jad.2019.06.042

Pena, M. y Extremera, N. (2010). Inteligencia emocional percibida en el profesorado de primaria y su relación con los niveles de burnout e ilusión por el trabajo (engagement). Revista de Educación, 359, 6046027. https://doi.org/10.4438/1988-592X-RE-2011359-109

Peña, J.E., Raso, P.C. y Ferrero, B.S. (2018). El síndrome de Burnout en los Docentes. Pirámide.

Powell, K. R., Mabry, J. L. y Mixer, S. J. (2015). Emotional intelligence: A critical evaluation of the literature with implications for mental health nursing leadership. Issues in Mental Health Nursing, 36(5), 346 $-356$. doi.org/10.3109/01612840.2014.994079 https://

Puertas Molero, P., Zurita, F., Ubago, J.L. y González , G. (2019). Influence of emotional intelligence and burnout syndrome on teachers well-being: A systematic review. Social Sciences, 8, 185. https:// doi.org/10.3390/socsci8060185

Pulido-Martos, M., Augusto-Landa, J.M., y Lopez-Zafra, E. (2016). Estudiantes de enfermería en prácticas clínicas: El rol de la inteligencia emocional en los estresores ocupacionales y bienestar psicológico. Index de Enfermería, 25(3), 215-219.

Pulido-Martos, M., Lopez-Zafra, E. y Augusto-Landa, J.M. (2013). Perceived emotional intelligence and its relationship with perceptions of effectiveness in negotiation. Journal of Applied Social Psychology, 43, 408-417. doi.org/10.1111/j.15591816.2013.01010.x

Pulido-Martos, M., Lopez-Zafra, E., Estévez-López, F. y Augusto-Landa, J.M. (2016). The moderator role of 
perceived emotional intelligence in the relationship between sources of stress and mental health in teachers. The Spanish Journal of Psychology, 19, 110. https://doi.org/10.1017/sjp.2016.8

Pulido-Martos, M., Luque-Reca, O., Augusto-Landa, J.M. y Ruiz, J. (2014). Inteligencia emocional, personalidad y actividad física en mayores institucionalizados. Revista Argentina de Clínica Psicológica, 23, 231-240.

Pulido-Martos, M., Luque-Reca, O., Segura-Jiménez, V., Álvarez-Gallardo, I.C., Soriano-Maldonado, A., Acosta-Manzano, P., Gavilán-Carrera, B., McVeigh, J.G., Geenen, R., Delgado-Fernández, M. y EstévezLópez, F. (2020). Physical and psychological paths toward less severe fibromyalgia: A structural equation model. Annals of Physical and Rehabilitation Medicine, 63(1), 46-52. https://doi.org/10.1016/ j.rehab.2019.06.017

Rivers, S.E., Brackett, M.A., Reyes, M.R., Elbertson, N.A. y Salovey, P. (2013). Improving the social and emotional climate of classrooms: A clustered randomized controlled trial testing The RULER Approach. Prevention Science, 14(1), 77-87. http:// dx.doi.org/10.1007/s11121-012-0305-2

Rodríguez-Espartal, N. y Lopez-Zafra, E. (2013). Programa emocional para presos por violencia de género (PREMOVIGE): Efectividad en variables cognitivas y conductuales. Psychosocial Intervention, 22(2), 115-123. https://doi.org/10.5093/in2013a14

Rutter M. (1987). Psychosocial resilience and protective mechanisms. American Journal of Orthopsychiatry, 57(3), 316-331. https://doi.org/10.1111/j.19390025.1987.tb03541.x

Salanova, M., Llorens, S. y García-Renedo, M. (2003). ¿Por qué se están quemando los profesores? Prevención, Trabajo y Salud, 28, 16-20.

Salanova, M., Llorens, S. y Martínez, I.M. (2019). Organizaciones saludables. Una mirada desde la psicología positiva. Aranzadi-Thompson Reuters.

Salovey, P., Bedell, B., Detweiler, J.B. y Mayer, J.D. (1999). Coping Intelligently: Emotional intelligence and the Coping Process. En C.R. Snyder (Ed.), Coping: The Psychology of What Works (pp. 141-164). Oxford University Press

Salovey, P. y Mayer, J.D. (1990). Emotional intelligence. Imagination, Cognition and Personality, 9(3), 185211. https://doi.org/10.2190/DUGG-P24E-52WK$6 \mathrm{CDG}$

Sánchez-Álvarez, N., Berrios, M.P. y Extremera, N.
(2020). A meta-analysis of the relationship between emotional intelligence and academic performance in secondary education: A multi-stream comparison. Frontiers in Psychology, 11, 1517. https:// doi.org/10.3389/fpsyg.2020.01517

Scheibe, S. y Carstensen, L.L. (2009). Emotional aging: Recent findings and future trends. The Journal of Gerontology Series B, 65B(2), 135-144. https:// doi.org/10.1093/geronb/gbp132

Schmitz, P.G. y Schmitz, F. (2012). Emotional intelligence and acculturation. Behavioral Psychology / Psicología Conductual, 20(1), 15-41.

Soto-Rubio, A., Giménez-Espert, M. y Prado-Gascó, V. (2020). Effect of emotional intelligence and psychosocial risks on burnout, job satisfaction, and nurses' health during the COVID-19 pandemic. International Journal of Environmental Research and Public Health, 17(21), E7998. https://doi.org/10.3390/ ijerph17217998

Sutton, R.E. (2004). Emotional regulation goals and strategies of teachers. Social Psychology of Education: An International Journal, 7(4), 379-398. https:// doi.org/10.1007/s11218-004-4229-y

Szczygiel, D.D. y Mikolajczak, M. (2018). Emotional intelligence buffers the effects of negative emotions on job burnout in nursing. Frontiers in Psychology, 9, 2649. https://doi.org/10.3389/fpsyg.2018.02649

Thomas, E., Moss-Morris, R. y Faquhar, C. (2006). Coping with emotions and abuse history in women with chronic pelvic pain. Journal of Psychosomatic Research, 60(1), 109-112. https://doi.org/10.1016/ j.jpsychores.2005.04.011

Tillmann, T., Krishnadas, R., Cavanagh, J. y Petrides, K.V. (2013). Possible rheumatoid arthritis subtypes in terms of rheumatoid factor, depression, diagnostic delay and emotional expression: An exploratory case-control study. Arthritis Research \& Therapy, 15(2), R45. https://doi.org/10.1186/ar4204

Torres Fúnez, E. y Lopez-Zafra, E. (2010). Diferencias en cultura del honor, inteligencia emocional y pensamientos distorsionados sobre las mujeres en reclusos y no reclusos. Boletín de Psicología, 100, 71-88.

Travers, C.J. (2001). Stress in teaching: Past, Present and Future. En J. Dunham (Ed.), Stress in the Workplace: Past, Present and Future (pp. 130-163). Whurr Publishers.

Tsirigotis, K. y Łuczak, J. (2016). Emotional intelligence of women who experience domestic violence. Psychiatric Quarterly, 87(1), 165-176. https:// 
doi.org/10.1007/s11126-015-9368-0

Updegraff, J.A. y Taylor, S.E. (2000). From Vulnerability to Growth: Positive and Negative Effects of Stressful Life Events. En J.H. Harvey y E.D. Miller (Eds.), Loss and Trauma: General and Close Relationship Perspectives (pp. 3-28). BrunnerRoutledge.

Van Heck, G.L. y Den Oudsten, B.L. (2008). Emotional Intelligence: Relationships to Stress, Health, and Well-being. En A. Vingerhoets, I. Nyklíček y J. Denollet (Eds.), Emotion Regulation: Conceptual and Clinical Issues (pp. 97-121). Springer Science + Business Media.

Van Middendorp, H., Lumley, M.A., Moerbeek, M., Jacobs, J.W., Bijlsma, J.W. y Geenen, R. (2010). Effects of anger and anger regulation styles on pain in daily life of women with fibromyalgia: A diary study. European Journal of Pain, 14(2), 176-182. https://doi.org/10.1016/j.ejpain.2009.03.007

Weissberg, R.P. y O'Brien, M.U. (2004). What works in school-based social and emotional learning programs for positive youth development. The Annals of the American Academy of Political and Social Science, 591(1), 86-97. https:// doi.org/10.1177/0002716203260093

Winters, J., Clift, R.J.W. y Dutton, D.G. (2004). An exploratory study of emotional intelligence and domestic abuse. Journal of Family Violence, 19(5), 255-267. https://doi.org/10.1023/ B:JOFV.0000042076.21723.f3

Zautra, A.J., Hall, J.S. y Murray, K.E. (2010). Resilience: A New Definition of Health for People and Communities. En J.W. Reich, A.J. Zautra y J.S. Hall (Eds.), Handbook of Adult Resilience (pp. 3-29). The Guilford Press.

Zunhammer, M., Halski, A., Eichhammer, P. y Busch, V. (2015). Theory of mind and emotional awareness in chronic somatoform pain patients. Plos One, 10(10), e0140016.

https://doi.org/10.1371/

journal.pone. 0140016 